\title{
Numerical Simulation of Abrikosov Vortex at Columnar Defect in Superconductor
}

\author{
V.A. Fedirko ${ }^{1, *}$, S.V. Polyakov ${ }^{2}$, A.L. Kasatkin ${ }^{3}$ and M.V. Fedirko ${ }^{4}$ \\ ${ }^{1}$ Moscow State Technological University "STANKIN", RU-127055, Moscow, Russia \\ ${ }^{2}$ Keldysh Institute of Applied Mathematics Russian Academy of Sciences, RU- 125047, Moscow, Russia \\ ${ }^{3}$ Kurdyumov Institute for Metal Physics, N.A.S. of Ukraine, UA-03142, Kiev, Ukraine \\ ${ }^{4} \mid$ Moscow Institute of Physics and Technology, RU- 141701, Dolgoprudny, Moscow Region, Russia
}

\begin{abstract}
We report a numerical modeling of single vortex depinning and its subsequent dynamics in HTS film with extended linear defects under the influence of the transport current. Numerical simulation of stable pinned vortex state and its escape from a linear defect has been performed. The non-stationary dynamics of vortex escape has been investigated and time-dependent solution for vortex displacement from the defect has been obtained. The delay effect in vortex escape process has been studied and the time delay has been estimated. The impact of processes being studied on electrodynamic properties of a superconductor has also been discussed. The dynamics of vortex escape from columnar pinning site described in the present work is important both for understanding of vortex dynamics and applying high-Tc superconductors with columnar defects.
\end{abstract}

\section{Introduction}

The superconducting state in hard superconductors, high-temperature superconductors (HTS) among them, holds until Abrikosov vortices, i.e., quantized lines of the magnetic flux, are fixed ("pinned") by specific material defects - pinning sites [1]. The model of an elastic string for Abrikosov vortex has proven highly useful for type-II superconductor and was shown to have large range of validity for in anisotropic and layered HTS with magnetic field $\boldsymbol{B}$ along the crystalline $c$ axis [2]. In our previous works [3-4] we have used that model for studying stationary states and critical current for a vortex at the potential well of extended linear defects - the latter are commonly considered as the most effective pinning sites in HTS films. Here we report on the attempt to an approach the problem of modelling vortex dynamics under the effect of time-dependent transport current. This paves the way for estimating their electrodynamic properties.

\section{Results and Discussion}

As in [3-4] we consider layered superconductor plate of thickness $d$ with linear defects oriented along the crystallographic $c$-axis, perpendicularly to plates surface, in the assumption that the magnetic field $B$ in the superconductor is low enough to neglect the intervortices interaction and believe that at low temperatures (formally at $T \rightarrow 0$ limit) all vortices are pinned by columnar defects. Due to very low mass density of a vortex one can limit with its "relaxation" dynamics described by the equation [5-8] related per vortex unit length:

$$
\eta \frac{\partial s}{\partial t}=P \frac{\partial^{2} s}{\partial z^{2}}+\phi_{0} \cdot j(t, z)-\frac{\partial U_{p}}{\partial s},
$$

where $s(t, z)$ is the vortex displacement from the linear defect axis ( $z$ - axis, $-d / 2<z<d / 2$ ) at time $t, P$ is the vortex line tension [2], $\phi_{0}$ is the magnetic flux quantum, $j(t, z)$ is the transport current density, $\eta$ - the viscosity coefficient, $U_{p}(s)$ - the pinning potential of a linear defect [8-10]. For the latter we use, as in [3-5) the Lorentzian-like form which is commonly employed to describe vortex pinning by columnar defects (see, e.g., [10-13]) and fit to Ginzburg-Landau theory (for details see [5]).

$$
U_{p}(s)=-U_{p 0} \frac{r_{p}^{2}}{r_{p}^{2}+s^{2}}
$$

We use dimensionless variables for numerical simulation with $z$, $s$ and $t$ normalized by $d / 2$, by $r_{p}$, and by $\bar{t}=\left(\eta d^{2} / 4 P\right)$ respectively. Then we come to the Neumann boundary problem (in view of the $\pm Z$ symmetry): 


$$
\begin{aligned}
& \frac{\partial s}{\partial t}=\frac{\partial^{2} s}{\partial z^{2}}-b \frac{s}{\left(1+s^{2}\right)^{2}}+a(t) \cdot \cosh (\alpha z), \\
& 0<z<1, \quad t>0 ; \\
& \frac{\partial s}{\partial z}(t, 0)=0, \quad \frac{\partial s}{\partial z}(t, 1)=0 ; \\
& s(0, z)=s_{0}(z), \quad 0<z<1 ;
\end{aligned}
$$

Here we put:

$$
a=\frac{\phi_{0} d^{2} j_{0}}{4 \operatorname{Pr}_{p}}, b=\frac{U_{p 0} d^{2}}{2 \operatorname{Pr}_{p}^{2}}, \alpha=\frac{d}{2 \lambda},
$$

and take into account that the transport current density $j(t, z)$ along the plate decays into the specimen interior from its surface value $j_{s}$ due to the Meissner effect [1]:

$$
j(z)=j_{0}(t) \cosh (z / \lambda), j_{0}=j_{s} / \cosh (d / 2 \lambda),
$$

$\lambda$ being the London penetration depth; the boundary condition at $z=1$ follows from the presumption of normal approach of vortex lines to the plates surfaces. The problem (3) is significantly non-linear due to the second term on the right hand of the equation of vortex motion.

To solve the problem numerically we apply the implicit finite-difference scheme and Newton iteration procedure to cope with the above non-linearity. The sweep procedure is used for realization of each iteration.

We start from the equilibrium vortex line state in the absence of transport current $s_{0}(z)=0$ and track its motion under the effect of quasi-step like increasing current pulse

$$
a(t)=a_{m}\left(1-e^{-t / \tau}\right)
$$

with variable pulse increment parameter $\tau$. We use our results of stationary vortex state and critical current modeling obtained earlier [3-5] to estimate the range for $a_{m}$ variation.

We have observed that when maximum pulse current is less than the critical current, hat is $a_{m}<a_{c r}$, the vortex at $t>\tau$ promptly reaches its stationary state coinciding with that found from static equation [3-5]. This is illustrated in Fig.1a, b where the results of numerical simulation of a vortex line evolution are presented for typical HTS film parameters $\alpha=3, b=5 \cdot 10^{3}$, $a_{c r}=353$, and for $a_{m}=300, \tau=0.05$, together with stationary vortex states from [1] in Fig. 1c: curve 7 in Fig. $1 \mathrm{~b}$ coincides with curve 3 in Fig.1c. With slow pulse increase, $\tau>>0.1$ (say, $\tau=1$ ), the vortex line evolves through the set of intermediate stationary states - that well correlate with the analytical estimation of relaxation time of a vortex string for Eq. (3) $\tau_{r}=1 / \pi^{2}$.

If $a_{m}>a_{c r}$, the depinning process starts at the moment $a(t)$ reaches $a_{c r}$ with runaway of the vortex edge from the defect at the plate surface and spreads inward the layer as shown in Fig 2 for $a_{m}=400$ and the other parameters as above. Such scenario was predicted in [35] and seems quite natural as the Lorentz force is strongest just at the surface of superconductor. As one can see in Fig. 3, there is even no external potential barrier at the surface when the transport current is close to its critical value so the vortex fraction near surface is held in the stationary state only by the string tension because its interior part is still in the potential well; hear by the external potential barrier we mean

$$
W_{e x t}=-\frac{b}{1+s^{2}}-a \cdot s \cdot \cosh (\alpha z) \text {. }
$$

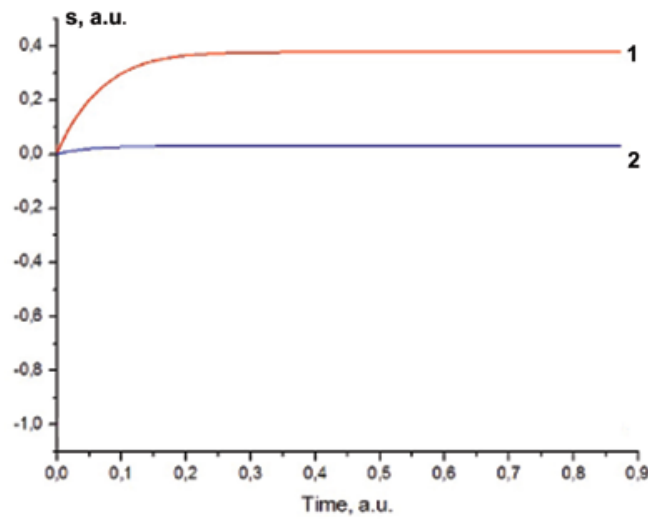

Fig.1a. Drift of the vortex edges under the effect of subcritical current pulse: $1-s(t, 1), 2-s(t, 0)$.

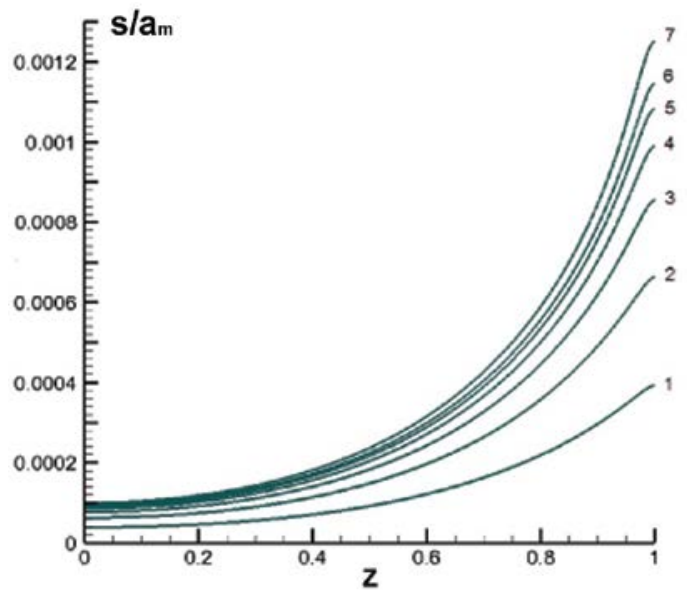

Fig. 1b. Vortex line evolution under the effect of subcritical current pulse, curves $1-7: t=0.025,0.050,0.075,0.100$, $0.125,0.150,0.375$

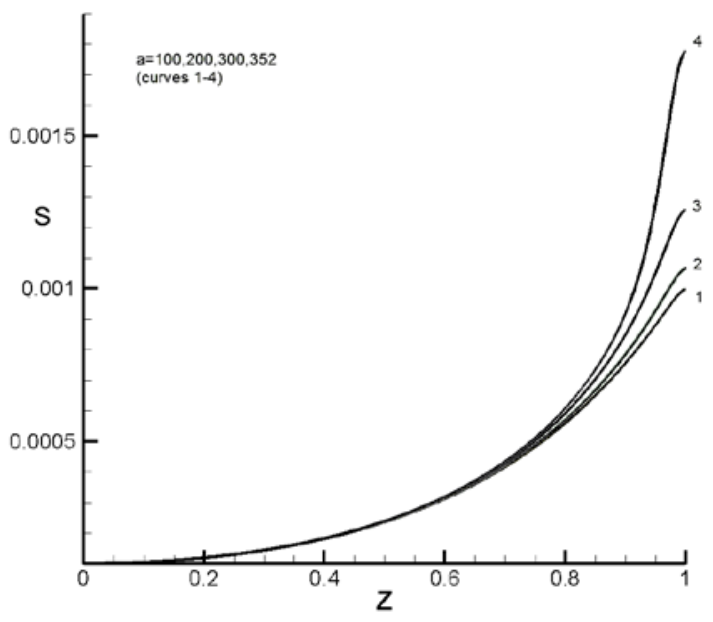

Fig. 1c. Stationary vortex lines for various subcritical transport currents [1] (here $s$ is divided by $a$ ). 


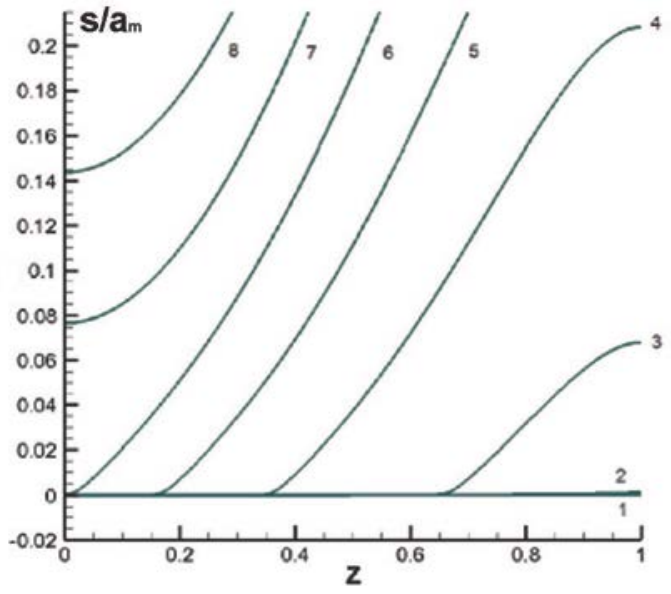

Fig. 2. Vortex line evolution under the effect of supercritical current pulse, curves $1-8: t=0.025,0.100,0.125,0.150$, $0.175,0.200,0.225,0.250$.

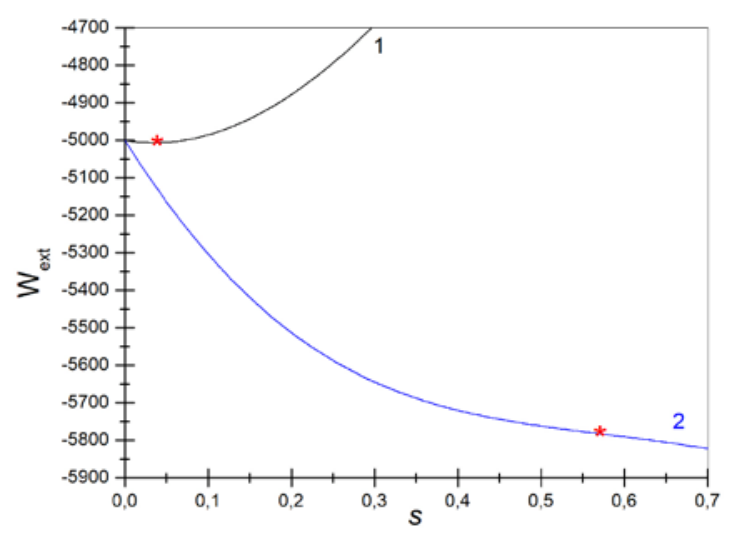

Fig. 3. Vortex edges locations (asterisk) on the external potential profile at near critical current $a=350$ : $1-z=0 ; 2-z=1$ $\left(a_{c r}=353\right)$

When the current reaches the critical value the string tension cannot hold the vortex edge any more and begins go away from the defect and thus launching the depinning.

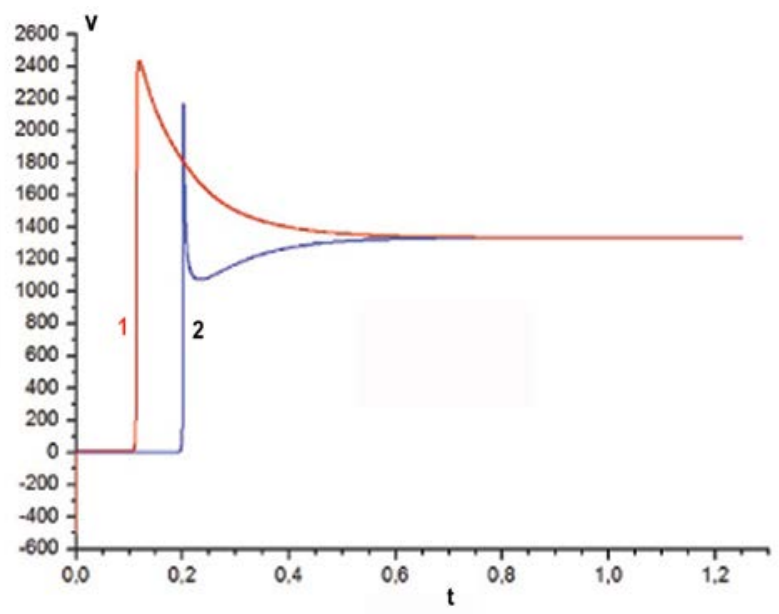

Fig. 4. Time dependence of vortex velocity at the surface (1) and in the middle (2) of the film.
The described scenario is clearly demonstrated by time dependence of the vortex velocity on the surface and in the middle of the superconductor film shown in Fig. 4. As the velocity of the vortex edge jump greatly just at the moment $t_{\mathrm{c}}$ the current reaches the critical value, the inner part of the vortex line remains motionless for some retention time $\tau_{r}$. Only at $t=t_{\mathrm{c}}+\tau_{r}$ its middle part tears off and all the vortex begins moving away from the defect. In some time the velocity relaxes to its stationary value uniform over all vortex string and being enough far away from the defect the vortex moves with constant velocity, its value $v_{\mathrm{s}}$ and the vortex line profile $\zeta(z)$ coincide with those found analytically in [3]:

$$
v_{s}=\frac{a_{m}}{\alpha} \sinh (\alpha) ; \quad \zeta(z)=\frac{a_{m}}{\alpha^{2}} \cdot[1-\cosh (\alpha z)]+\frac{a z^{2}}{2 \alpha} \sinh (\alpha)
$$

Below in Fig. 5 the time dependence of vortex drift is also presented for illustration.

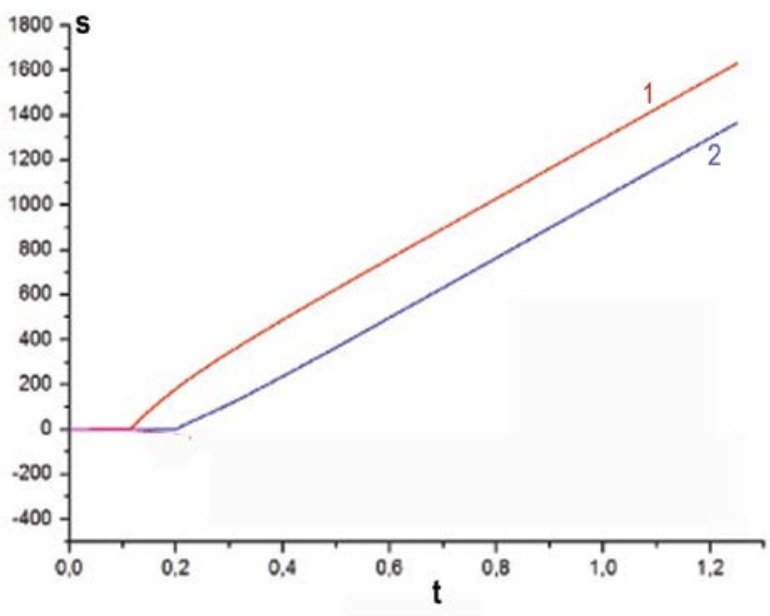

Fig. 5. Time dependence of vortex drift: $1-s(t, 1), 2-s(t, 0)$.

We estimate the retention time $\tau_{r}$ as time difference between velocity peaks of Fig. 4 . The dependence of $\tau_{r}$ on $\alpha$ for typical values $d>\lambda$ is presented in Fig. 6 .

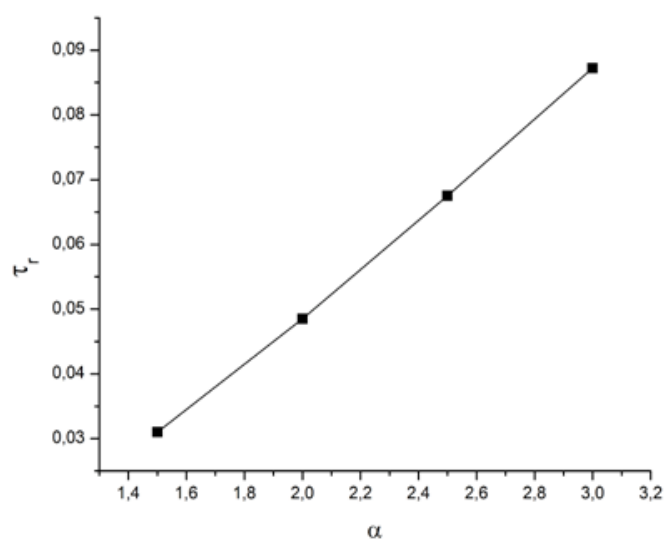

Fig. 6. Dependence of retention time $\tau_{r}$ on $\alpha$.

As one could expect retention gets shorter with $\alpha$ decreasing since the current density and accordingly the 
Lorentz force gets more uniform over the plate (5). The estimation gives the typical value of normalizing time $\bar{t} \in 10^{8} c$ for HTS REBCO material so we can estimate the retention time $\tau_{\gamma} \in\left(10^{-10} \div 10^{-9}\right)$ for $\alpha \in(1 \div 4)$.

These results imply some new physics which concerns vortex dynamics in type-II superconductors with columnar pinning sites, important both for understanding both of mixed state dynamics and of related electrodynamic properties in these superconductors as well as for their practical application. For the first time we introduce and demonstrate the existence of retention time $\tau_{r}$ for the process of vortex depinning from an extended linear defect, as it is demonstrated in Figs. 4-6. This delay in vortex depinning from columnar pinning sites under the varying Lorentz force should manifests itself in different electrodynamic properties of such superconductors and also leads to some unexpected features of their behavior under the ac or pulse current action, which are not described by the classical theories of the mixed state dynamics in type-II superconductors [8-10]. For instance, penetration of magnetic flux inside type-II superconductor settled in a varying external magnetic field proceeds via vortex diffusion from the specimen edges to its interior and in general can be described by the nonlinear and non-local diffusion equation for electric and/or magnetic field components inside the superconductor $[8,13]$. Solution of this equation gives, e.g., the specific logarithmic law for the magnetic moment decay $\boldsymbol{M}(t)$, dependent on some intrinsic parameters which characterize superconducting material and conditions of experiment - e.g., on the position of vortex ensemble during experiment on the $(H, T)$ - phase diagram.

For superconductors with columnar defects an account for the retention time $\tau_{r}$ of vortex escape from these pinning sites in some cases will significantly change the solution of the nonlinear diffusion equation mentioned above, as well as corresponding interpretation of experimental results on measurements of $\boldsymbol{M}(t)$ dependencies during flux creep experiments. One can expect some retard of the $\boldsymbol{M}(t)$ relaxation caused by the retention time $\tau_{r}$. That kind of vortex escape retention from columnar pinning sites probably should significantly affect vortex dynamics in rapidly varying magnetic field (comparatively to that in common superconductors with point-like vortex pinning sites, where $\tau_{r}$ goes to zero). Such experiments in rapidly varying magnetic fields now are of great interest [14,15]. Also the delay in vortex depinning from columnar defects discussed in the present work is important for operation of current switch devices (see, e.g. [16,17]).

\section{Conclusion}

We have studied numerically single vortex depinning and its subsequent dynamics in HTS film with extended linear defects under the effect of the Lorentz force caused by the transport current. Numerical simulation of stable pinned vortex state and its escape from linear defect has been performed. The non-stationary dynamics of vortex depinning process is studied and timedependent scenario of vortex escape from the defect is obtained. Delay effect in vortex escape process at critical current is demonstrated and the time delay is estimated. Corresponding effects on electrodynamic properties of a superconductor are discussed. The dynamics of vortex escape from columnar pinning site described in the present work is important both for understanding of vortex dynamics and applications of high-Tc superconductors with columnar defects.

The work was partially supported by the Russian Foundation for Basic Research, projects No. 17-01-00973-a and 20-0700790-a. We also thank the Center of Collective Use of MSUT "STANKIN" for providing resources.

\section{References}

1. Abrikosov, Fundamentals of the Theory of Metals, (Elsevier, Amsterdam, 1988)

2. E.H. Brandt, Phys. Rev. Lett. 69, 1105 (1992)

3. V.A. Fedirko, S.V. Polyakov, A.L. Kasatkin, Mathem. Models Compt. Simul. 6, 408 (2014)

4. V.A. Fedirko, A.L. Kasatkin, S.V. Polyakov, Phys. Met. Metallogr, 117(9), 864, (2016)

5. V.A. Fedirko, A.L. Kasatkin, and S.V. Polyakov, JLTP, 192(5), 359, (2018)

6. J.I. Gittleman and B.Rosenblum, Phys. Rev. Lett. 16, 734 (1966)

7. M.W. Coffey and J.R. Clem, PRL,67, 386, (1991)

8. E.H. Brandt, Rep. Progr. Phys. 58, 1465 (1995)

9. Tinkham, M. Introduction to Superconductivity (McGraw-Hill, 1996) ISBN-0-07-064878-6

10. G.M. Blatter, M.V. Feigel'man, V.B. Geshkenbein, A.I. Larkin, V.M. Vinokur, Rev. Mod. Phys. 66, 1125 (1994)

11. E.A. Pashitskii, V.I. Vakaryuk, Fiz. Nizk Temp. 28, 16 (2002)

12. A.V. Lopatin, V.M. Vinokur, PRL. 92, 067008 (2004)

13. A. Gurevich, Int. J. Modern Phys. B, 9, 1045 (1995)

14. L. Embon, Y. Anahory, Ž.L. Jelić, E.O. Lachman, Y. Myasoedov, M.E. Huber, G.P. Mikitik, A.V. Silhanek, M.V. Milošević, A. Gurevich, and E. Zeldov, Nature Commun. 8, 85 (2017)

15. M. Leroux, F. F. Balakirev, M. Miura, K. Agatsuma, L. Civale, and B. Maiorov, Phys. Rev. Applied 11, 054005 (2019)

16. V.F. Solovyov and Q.Li, Appl. Phys. Lett. 103, 032603 (2013)

17. L. Zhang, X. Yan, X. Jia, J. Chen, L. Kang, and P. Wu, Appl. Phys. Lett. 110, 072602 (2017) 